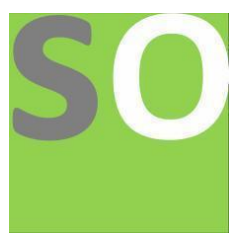

Article title: Building Information Modeling (BIM)

En Proyectos de Infraestructura Civil

Authors: RENZO APAZA CUTIPA[1], ROY ANAHUA MAMANI[2]

Affiliations: Escuela de posgrado/Universidad Nacional del Altiplano, Ciudad Universitaria, Puno, Puno, Perú[1]

Orcid ids: 0000-0002-7089-2812[1]

Contact e-mail: renzo@unap.edu.pe

License information: This work has been published open access under Creative Commons Attribution License http://creativecommons.org/licenses/by/4.0/, which permits unrestricted use, distribution, and reproduction in any medium, provided the original work is properly cited. Conditions, terms of use and publishing policy can be found at https://www.scienceopen.com/.

Preprint statement: This article is a preprint and has not been peer-reviewed, under consideration and submitted to ScienceOpen Preprints for open peer review.

DOI: 10.14293/S2199-1006.1.SOR-.PPATOPR.v1

Preprint first posted online: 28 March 2021

Keywords: BIM, infraestructura civil, modelado de información de edificación, proyectos, civil infrastructure, building information modeling 


\title{
BUILDING INFORMATION MODELING (BIM) EN PROYECTOS DE INFRAESTRUCTURA CIVIL
}

\section{BUILDING INFORMATION MODELING (BIM) IN CIVIL INFRASTRUCTURE PROJECTS}

\author{
APAZA CUTIPA RENZO,Renzo (renzo@unap.edu.pe)* \\ *Docente de la Facultad de Ingeniería Estadística e Informática, UNA - Puno \\ ANAHUA MAMANI, Roy Wiley (ranahua@unap.edu.pe)** \\ *Estudiante de la Maestría en Ingeniería Civil con mención en Gerencia de la Construcción, UNA - Puno
}

\section{RESUMEN}

Una de las necesidades más imperantes de la industria de la construcción está referida a la mejora de los rendimientos de los procesos de construcción, siendo una de las tendencias más significativas aplicadas para este propósito el modelado de información de construcción (BIM - Building Information Modeling). En la presente revisión se muestran los beneficios de su aplicación en los proyectos de construcción, el conocimiento de BIM desde una visión general del estado del arte. Y finalmente se presenta la descripción general, la aplicación teórica práctica, conclusiones y recomendaciones.

Palabras clave: BIM, infraestructura civil, modelado de información de edificación, proyectos.

\section{ABSTRACT}

One of the most prevailing needs of the construction industry is related to the improvement of the performance of construction processes, one of the most significant trends applied for this purpose is the modeling of construction information (BIM - Building Information Modeling) . This review shows the benefits of its application in construction projects, the knowledge of BIM from an overview of the state of the art. And finally the general description, the practical theoretical application, conclusions and recommendations are presented.

Keywords: BIM, civil infrastructure, building information modeling, projects. 


\section{INTRODUCCIÓN}

En Perú la ejecución de obras públicas sufre retrasos asociados a diferentes factores como la planificación, ejecución presupuestaria, transporte, logística en general; situaciones que generan un malestar generalizado en la población y lo más importante sobrecosto durante todas las etapas del ciclo de vida de la ejecución de obras (Álvaro León, 2019)(Flórez Cardoso, 2017)(Gamarra Barreros, 2014). Pero este problema no solo propio de nuestro país a nivel internacional, en relación a esto algunas obras establecen un sobrecoste medio del 12,22\% en proyectos de construcción en ingeniería(Love et al., 2013)(Plebankiewicz, 2018).

Los factores asociados a las pérdidas son diversos, como las causas de origen humano personas involucradas en el proceso, donde la falta de comunicación es una constante que aparece como consecuencia de la concurrencia de empresas y participantes en la fase de construcción, el clima de competencia, y rigidez de la información (Andújar-Montoya et al., 2015). Circunstancias o eventos que no permiten la llegada oportuna de la información a todas las partes interesadas o que no llegue a tiempo, lo que genera cuellos de botella, trabajos innecesarios y reelaboraciones (Alshawi \& Ingirige, 2003). Por lo regular un porcentaje de hasta el 30\% son tareas de retrabajo(Aziz \& Hafez, 2013), afectando el presupuesto y la planificación del proyecto(Hwang et al., 2009). (Akinci et al., 2006) y (Josephson \& Hammarlund, 1999) indica que del 6 al 12\% de los costes de construcción se desperdician en la reparación de componentes defectuosos detectados tarde durante la fase de construcción, y se agrava por la falta de motivación y participación de empresas subcontratadas y trabajadores propios. Además, las causas de origen humano se ven reforzadas por la alta dependencia del sector de la construcción de tareas y procedimientos manuales que podrían automatizarse mediante el uso de tecnología avanzada. Sin embargo, la escasez y brecha tecnológica es una constante en el sector que contribuye al ineficiente desempeño general de la industria de la construcción, promoviendo nuevamente la hermeticidad de la información, duplicidades y errores y en última instancia falta de transparencia durante el proceso constructivo(Andújar-Montoya et al., 2017). Si bien la tecnología representa uno de los principales aliados de las empresas para optimizar los recursos, la utilización de estos avances tecnológicos en la industria de la construcción sigue siendo bastante baja(Choo \& Tommelein, 2001). Además, son también destacables las causas inherentes a los proyectos de construcción derivadas de la complejidad, incertidumbre y variabilidad del propio proceso constructivo (Andújar-Montoya et al., 2015). (Abdelhamid \& Everett, 2002) indica además que las causas se pueden categorizar como retrasos en la entrega de materiales y equipos, errores de diseño, cambios en los pedidos, averías y mal 
funcionamiento de herramientas, ausencias laborales, efectos ambientales, accidentes y demandas físicas del trabajo que afectan la productividad. Para resolver esta problemática en la Unión Europea(UE) por ejemplo se introdujeron medidas para digitalizar el proceso constructivo, proponiendo el uso de BIM, en base a la experiencia que tenían otros países de la UE y que muchos investigadores han analizado (Porwal \& Hewage, 2013). Como resultado de lo anterior, es necesario un replanteamiento de las prácticas y procedimientos utilizados hasta ahora, siendo ineludible la definición de nuevos lineamientos de acción para una mayor eficiencia en los proyectos de construcción. En función de lo indicado se examina la aplicación de las tendencias más crecientes del sector en este momento con el fin de mejorar la eficiencia del proceso constructivo, tanto en términos de tiempo como de costo, abordando las metodologías de trabajo colaborativo, el modelado de información de construcción (en adelante BIM). BIM es una tecnología emergente que proporciona una solución integral para la gestión de proyectos de construcción a lo largo de su ciclo de vida, desde la definición de los objetivos del proyecto hasta el cumplimiento de su vida útil, centralizando toda la información del proyecto en un modelo de información digital accesible para todos los stakeholders(GalianoGarrigós \& Andújar-Montoya, 2018).

El estado Peruano, el 28 de julio de 2019, ha aprobado el Plan Nacional de Competitividad y Productividad, mediante el Decreto Supremo N. 237-2019-EF, establece como Medida de Política 1.2 la adopción progresiva de la metodología BIM en el sector público. Asimismo, mediante el Decreto Supremo N. 289-2019-EF se aprueba las disposiciones para la incorporación progresiva de BIM en la inversión pública de las entidades y empresas públicas sujetas al Sistema Nacional de Programación Multianual y Gestión de Inversiones. Este Plan BIM Perú es un instrumento de gestión que define los objetivos y acciones estratégicas para la utilización progresiva de la metodología BIM en las inversiones públicas al año 2030. El cual nace con la finalidad de garantizar e incrementar una adecuada ejecución de las inversiones públicas en edificaciones e infraestructura, mejorando la calidad y eficiencia de los proyectos desde su diseño, durante su diseño, construcción, operación y hasta su mantenimiento. Teniendo como objetivo principal, Garantizar una adecuada ejecución de las inversiones de edificaciones e infraestructura desde el sector público, incorporando procesos, metodologías y tecnologías de información a lo largo del ciclo de inversión. Asimismo, el Plan BIM Perú busca fomentar un articulado proceso de adopción por parte del sector público, acompañado del sector privado y la academia (https://www.mef.gob.pe/es/?option=com_content\&language=es$\underline{\text { ES\&Itemid=102596\&lang=es-ES\&view=article\&id=5898). }}$. 


\section{EL MODELO DE BIM}

La mejora tecnológica más extendida en el sector es la implantación de BIM como tendencia creciente que aporta una solución integral para la gestión de proyectos de construcción, a lo largo de su ciclo de vida desde la definición de los objetivos del proyecto hasta el cumplimiento de su vida útil(Galiano-Garrigós \& Andújar-Montoya, 2018). Es el nuevo estándar tecnológico basado en una mejora de la gestión de datos de edificios que está renovando el mercado de la construcción. Esta información, recopilada durante el proceso de construcción, se convierte en un valor agregado durante todo el ciclo de vida del edificio. Esto se logra porque BIM se basa en una representación digital del proceso de construcción que facilita el intercambio y la interoperabilidad de la información en formato digital. Paralelamente, BIM se está introduciendo progresivamente en el ciclo de vida del proyecto. Así en Europa los gobiernos nacionales vienen adecuando sus procedimientos constructivos a la publicación por parte de la Unión Europea (UE) de la Directiva Europea de Contratación Pública, que promueve el uso de herramientas electrónicas como BIM en proyectos de construcción financiados con fondos públicos de la UE. Pero aunque algunos países como Reino Unido, Dinamarca, Holanda, Noruega y Finlandia ya exigen BIM para obras públicas, hay otros países, como España, donde la conciencia y la adopción entre los actores de la construcción se está extendiendo actualmente (Muñoz, s. f.), y la implementación no ha comenzado para la administración y aún se encuentra en una etapa inicial sobre todo para las pymes.

Hay varios autores que se han centrado en la aceptación e implementación de BIM en el sector (Kensek, 2014), (Succar, 2009), (Azhar et al., 2012), (Bryde et al., 2013), (Miettinen \& Paavola, 2014). El uso de tecnologías BIM durante las fases de diseño y construcción evita

errores de diseño permitiendo la visualización y por tanto conflictos, interferencias y detecciones de choques (Khanzode et al., 2008), logrando ahorros de hasta un $10 \%$ del valor del contrato a través de estas detecciones de choques (Azhar, 2011). De hecho, se ha demostrado que BIM reduce los errores y omisiones de documentos hasta en un 52\%, también puede reducir los reprocesos hasta en un $48 \%$ y reducir el tiempo de ciclo de flujos de trabajo específicos en un 39\% y el tiempo de ciclo de flujos de trabajo específicos hasta en un 39\% (McGraw Hill Construction, 2012), (Zhang et al., 2018). Además, son notables los beneficios relacionados con la reducción de costos y el control a lo largo del ciclo de vida del proyecto (Bryde et al., 2013) ya que BIM proporciona información predecible con respecto a la cantidad, costo, cronogramas y materiales(Chen et al., 2013). En la siguiente figura se observa la representación del modelo BIM de una edificación modelada en los softwares Autodesk Revit 2016 y 
Navisworks Manage 2016, a través de los cuales, se puede visualizar los conflictos, interferencias y detecciones durante las fases de diseño y previo a la construcción.
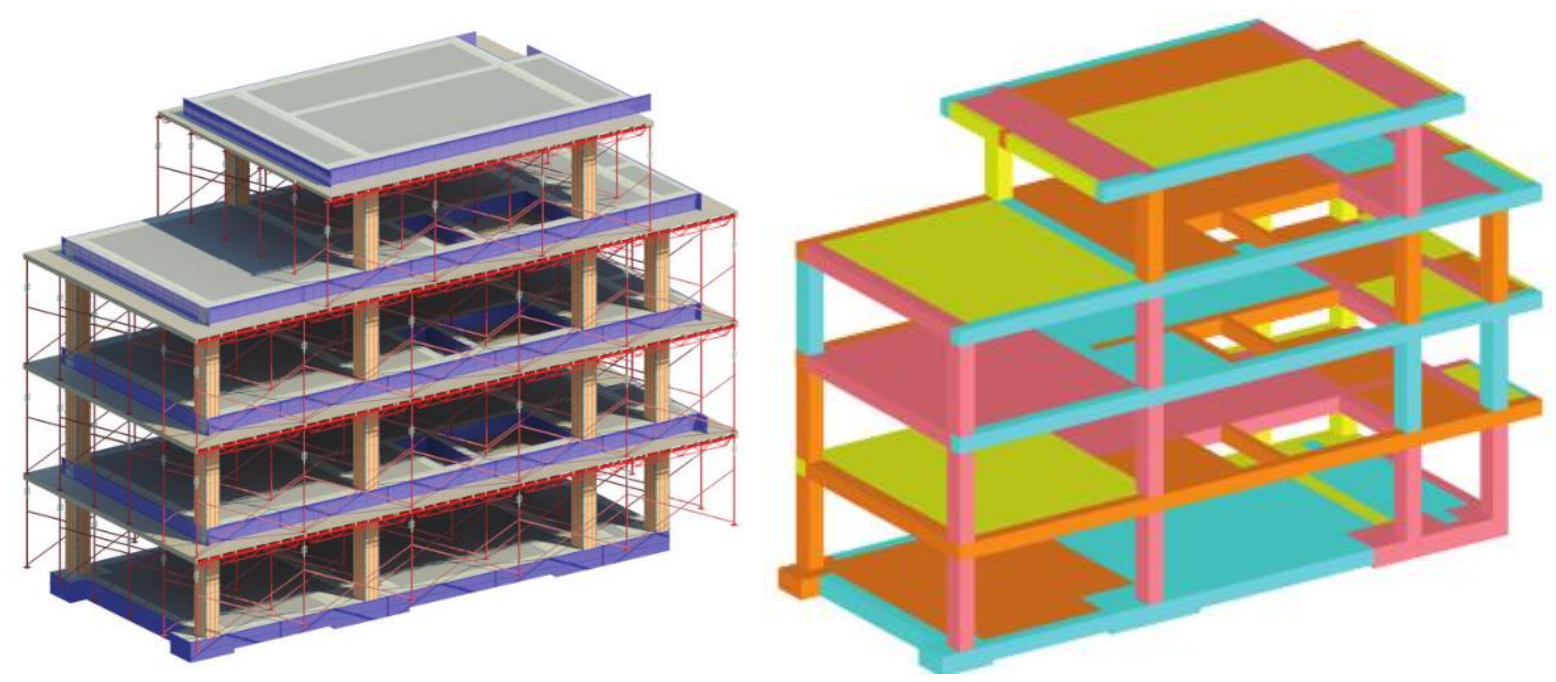

Figura 1. Modelo BIM 3D

Fuente: (Bohórquez-Castellanos et al., 2018)

Además, BIM crea y pone a disposición información de forma temprana, este hecho junto con la ubicuidad de la tecnología móvil que facilita el acceso a la información del sitio en cualquier momento y desde cualquier lugar (Garita-Araya, 2013), acorta la brecha entre la disponibilidad de información y los tiempos de respuesta. Además, BIM facilita el flujo de información ya que los participantes tienen una comprensión clara de su rol y responsabilidad en un proyecto alineando las expectativas, y aumenta la transparencia, ya que muestra cómo integrar las mejores prácticas y capturar el intercambio de conocimientos de un proyecto a otro, promoviendo la mejora continua(Rogério dos Santos Alves; Alex Soares de Souza, 2014).

Compatibilidad con la metodología tradicional: Al igual que BIM permite realizar innovadores Modelos 3D, es posible también no alejarse en exceso de los tradicionales proyectos en 2D, porque tenemos que ser conscientes que no todos los profesionales de la construcción están habituados a trabajar con esta metodología que todavía está en pleno desarrollo e implantación dentro del mundo de las obras civiles. Del mismo modo, podemos trabajar simultáneamente con vistas $2 \mathrm{D}$ y $3 \mathrm{D}$, lo que nos facilita una experiencia más completa y nos ayuda a tener una mejor concepción global del proyecto (Manuel Dorado Rasero, 2020). 


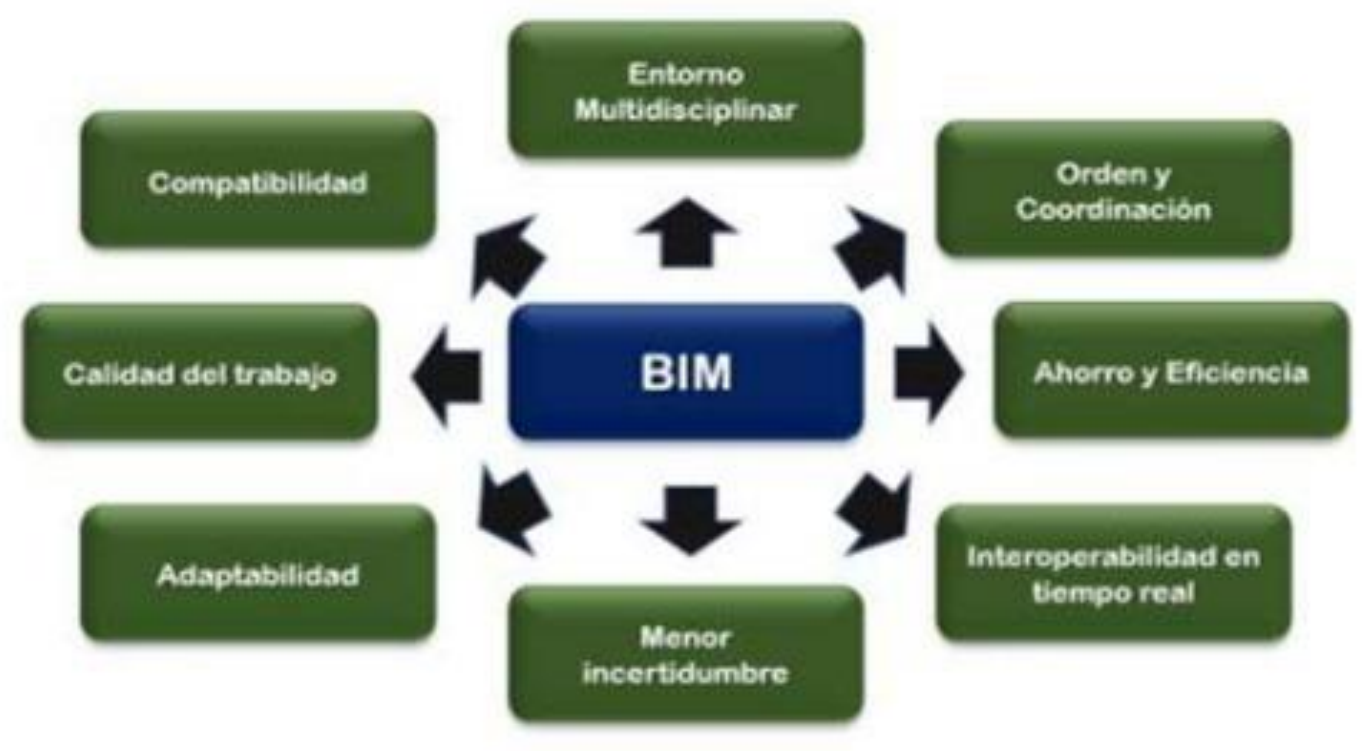

Figura 2. Ventajas de la Aplicación de la Metodología BIM Fuente: (Manuel Dorado Rasero, 2020)

\section{METODOLOGÍA}

Según (de Carvalho et al., 2017) el análisis de contenido es una técnica de investigación que permite la recolección y clasificación de información con el fin de identificar tendencias. La principal característica del análisis de contenido es que es un método que combina intrínsecamente la observación y la interpretación de los datos o análisis de datos. El presente trabajo se centra en un análisis de contenido sobre BIM. Se incluyeron trabajos basados en esta en revistas científicas y en los congresos internacionales más representativos.

\section{ANÁLISIS Y DISCUSIÓN}

Dados los diversos resultados positivos para cada etapa del ciclo de vida del proyecto cuando se implementa BIM, se recomienda la implementación de BIM en todas las etapas de los proyectos de construcción. La teoría existente ha identificado varios beneficios que se producen para el inicio, diseño, producción, mantenimiento y descomposición. El uso de BIM brinda un beneficio más significativo para cada etapa del proyecto. En la parte inicial, BIM se utiliza como herramienta conceptual (Azhar et al., 2008).

Además, la construcción de modelos 3D y la captura de todos los datos relevantes conduce a varios resultados positivos en todas las etapas del ciclo de vida del proyecto y para todas las partes interesadas. Para lograr los beneficios de BIM, es obligatorio una transición, así como una mentalidad técnica específica. La ausencia de dirección para este desarrollo y la cantidad reducida de estudios arraigados en la realidad para ayudar a las empresas en su adopción 
representan un inconveniente y una barrera importantes para el uso extensivo entre la industria de la construcción civil(Marshall-Ponting et al., 2009). Por lo tanto, los gerentes de proyecto capacitados en BIM deben ser asignados como líderes de proyecto con más frecuencia, ya que es probable que implementen BIM en un proyecto y generen conciencia. También es vital comprender cómo opera el proceso de notificación con el modelo para garantizar que cualquier alteración en el diseño general haya sido entendida, probada y confirmada completamente por otras partes del contrato. Una posible respuesta a este problema es que el equipo del proyecto adquiera una póliza de seguro de proyecto única en particular. Esto podría resolver los asuntos contractuales entre las partes y eliminar la dificultad de establecer las responsabilidades relevantes de los consultores. La utilización de BIM probablemente aumente significativamente debido a un impulso para que los proyectos públicos utilicen dichas tecnologías (Sabol, 2008). La calidad de la planeación determina el éxito fracaso de la ejecución, operación y mantenimiento de proyectos, con la metodología conocida como Building Information Modeling (BIM) es posible mejorar el resultado de la planeación mediante la implementación de modelos digitales en la toma de decisiones.(Prieto-Tibaduiza et al., 2019)

Otro punto importante es que además de mejorar la tecnología, BIM facilita una toma de decisiones más efectiva en las etapas de diseño y producción del proyecto. El uso de BIM reduce los costos de diseño y puede acelerar la entrada al mercado, al tiempo que reduce la ambigüedad e integra múltiples disciplinas, que incluyen datos, diseño y documentación. BIM beneficia a los arquitectos, ya que elimina el trabajo de verificación manual y facilita la toma de decisiones y la ejecución rápidas en diversas tareas del proyecto. En la etapa de operación, la salida informativa de BIM abarca parámetros de desempeño del proyecto, tanto en las operaciones del proyecto como en el aspecto económico del mismo. Estos parámetros permiten la toma de decisiones informada y ayudan a garantizar resultados positivos del proyecto. Cuando se utiliza de manera efectiva, BIM también podría disminuir el tiempo de construcción, reducir los costos generales, respaldar la documentación digital del proyecto e incluso asegurarse de que las relaciones entre las partes interesadas clave sean las esperadas.

Los propietarios y gerentes del proyecto deben elaborar un plan detallado y objetivos bien definidos con respecto a BIM que permitan una adopción e implementación más efectivas.

La investigación actual identificó factores y resultados relevantes de BIM y establece un marco para un estudio futuro. Sobre la base de la revisión de factores, se debe construir y probar empíricamente un modelo conceptual que pruebe la relación entre varios resultados BIM y los 
factores que impactan la implementación. Este tipo de estudios cualitativos ayudaría a llenar el vacío, ya que la investigación predominante en BIM es principalmente de naturaleza cualitativa (Son et al., 2015).

\section{CONCLUSIONES}

El uso de BIM y sus beneficios han demostrado ser invaluables para la ejecución de los proyectos de construcción. Las ventajas se obtienen mediante la colaboración eficaz de los miembros del equipo durante el proceso constructivo de los proyectos, mediante el uso de tecnologías BIM. No obstante, estas herramientas tienen sus desventajas que requieren una mayor consideración tanto por parte de los teóricos como de los profesionales de BIM. Los directores de proyectos, diseñadores, arquitectos y otras partes involucradas que forman parte de los proyectos de construcción deben adquirir nuevos conocimientos e innovaciones.

En particular, BIM y sus herramientas están teniendo una buena acogida en proyectos de construcción. Los valores paramétricos y los indicadores económicos disponibles en BIM a lo largo del proceso de diseño, construcción, operación y mantenimiento permiten la toma de decisiones objetivas, lo que se traduce en varios beneficios. La configuración y coordinación a través de la utilización de BIM minimiza los riesgos que ocurren durante la etapa de construcción del proyecto. Se mostró como BIM es beneficioso para los proyectos de construcción. BIM produce resultados de construcción de mejor calidad y aumenta la eficiencia de los procesos durante todas las etapas del proyecto. Se pueden utilizar diferentes tecnologías BIM para actualizar BIM en proyectos.

También se identificó otro escenario que corresponde a las facilidades que se puede obtener aplicando el modelo BIM en las diferentes etapas de los proyectos; planificación, diseño, construcción y hasta operación y mantenimiento. Se muestra que el éxito de la implementación de BIM depende de la constante actualización de la información. Y es necesario que los profesionales se familiaricen con esta metodología, no como una herramienta; sino como un sistema para generar un modelo de información constructiva de una obra. BIM en sí no es un archivo Revit, ni una aplicación en la nube, ni algo que compramos en una tienda, el BIM es una disciplina y una serie de procesos hechos con diversas herramientas.

Por último las tecnologías BIM todavía tienen inconvenientes y desafíos y requieren más mejoras. En general, BIM es un concepto revolucionario en la industria de la construcción civil, sin embargo, aún no ha alcanzado niveles suficientes de adopción. 


\section{BIBLIOGRAFIA}

Abdelhamid, T. S., \& Everett, J. G. (2002). Physical Demands of Construction Work : a Source of Workflow Unreliability. Proceedings IGLC-10.

Akinci, B., Boukamp, F., Gordon, C., Huber, D., Lyons, C., \& Park, K. (2006). A formalism for utilization of sensor systems and integrated project models for active construction quality control. Automation in Construction. https://doi.org/10.1016/j.autcon.2005.01.008

Alshawi, M., \& Ingirige, B. (2003). Web-enabled project management: An emerging paradigm in construction. En Automation in Construction. https://doi.org/10.1016/S09265805(03)00003-7

Álvaro León, J. L. (2019). Identificación de factores que originan sobrecostos en el sector de construcción en proyectos corporativos en el Perú, al aplicar el modelo SCOR.

Andújar-Montoya, M. D., Gilart-Iglesias, V., Montoyo, A., \& Marcos-Jorquera, D. (2015). A construction management framework for mass customisation in traditional construction. Sustainability (Switzerland). https://doi.org/10.3390/su7055182

Andújar-Montoya, M. D., Marcos-Jorquera, D., García-Botella, F. M., \& Gilart-Iglesias, V. (2017). A context-driven model for the flat roofs construction process through sensing systems, internet-of-things and last planner system. Sensors (Switzerland). https://doi.org/10.3390/s17071691

Azhar, S. (2011). Building information modeling (BIM): Trends, benefits, risks, and challenges for the AEC industry. Leadership and management in engineering, 11(3), 241-252.

Azhar, S., Khalfan, M., \& Maqsood, T. (2012). Building information modelling (BIM): now and beyond. Construction Economics and Building, 12(4), 15-28.

Azhar, S., Nadeem, A., Mok, J. Y. N., \& Leung, B. H. Y. (2008). Building Information Modeling (BIM): A new paradigm for visual interactive modeling and simulation for construction projects. Proc., First International Conference on Construction in Developing Countries, 1, 435-446.

Aziz, R. F., \& Hafez, S. M. (2013). Applying lean thinking in construction and performance improvement. Alexandria Engineering Journal. https://doi.org/10.1016/j.aej.2013.04.008

Bohórquez-Castellanos, J. J., Porras-Díaz, H., Sánchez-Rivera, O. G., \& Mariño-Espinel, M. 
C. (2018). Planificación de recursos humanos a partir de la simulación del proceso constructivo en modelos BIM 5D. Entramado, 14(1), 252-267. https://doi.org/10.18041/entramado.2018v14n1.27141

Bryde, D., Broquetas, M., \& Volm, J. M. (2013). The project benefits of building information modelling (BIM). International Journal of Project Management. https://doi.org/10.1016/j.ijproman.2012.12.001

Chen, S. M., Griffis, F. H., Chen, P. H., \& Chang, L. M. (2013). A framework for an automated and integrated project scheduling and management system. Automation in Construction. https://doi.org/10.1016/j.autcon.2013.04.002

Choo, H. J., \& Tommelein, I. D. (2001). Requirements and Barriers To Adoption of Last Planner Computer Tools. Ninth Annual Conference of the International Group for Lean Construction.

De Carvalho, A. C. V., Granja, A. D., \& da Silva, V. G. (2017). A systematic literature review on integrative lean and sustainability synergies over a building's lifecycle. Sustainability (Switzerland). https://doi.org/10.3390/su9071156

Flórez Cardoso, P. (2017). Factores determinantes de sobrecostos en obras públicas liquidadas por la Municipalidad Provincial del Cusco.

Galiano-Garrigós, A., \& Andújar-Montoya, M. D. (2018). Building information modelling in operations of maintenance at the university of alicante. International Journal of Sustainable Development and Planning. https://doi.org/10.2495/SDP-V13-N1-1-11

Gamarra Barreros, R. H. (2014). Evaluación de sobrecostos de obras civiles del proyecto red de transmisión microondas Yurimaguas-Iquitos.

Garita-Araya, R. A. (2013). Tecnología Móvil: desarrollo de sistemas y aplicaciones para las Unidades de Información. e-Ciencias de la Información. https://doi.org/10.15517/eci.v3i2.10654

Hwang, B.-G., Thomas, S. R., Haas, C. T., \& Caldas, C. H. (2009). Measuring the Impact of Rework on Construction Cost Performance. Journal of Construction Engineering and Management. https://doi.org/10.1061/(asce)0733-9364(2009)135:3(187)

Josephson, P. E., \& Hammarlund, Y. (1999). Causes and costs of defects in construction a study of seven building projects. Automation in construction. https://doi.org/10.1016/S0926- 
5805(98)00114-9

Kensek, K. M. (2014). Building information modeling. En Building Information Modeling. https://doi.org/10.4324/9781315797076

Khanzode, A., Fischer, M., \& Reed, D. (2008). Benefits and lessons learned of implementing Building Virtual Design and Construction (VDC) technologies for coordination of Mechanical, Electrical, and Plumbing (MEP) systems on a large Healthcare project. Electronic Journal of Information Technology in Construction.

Love, P. E. D., Wang, X., Sing, C., \& Tiong, R. L. K. (2013). Determining the Probability of Project Cost Overruns. Journal of Construction Engineering and Management. https://doi.org/10.1061/(asce)co.1943-7862.0000575

Marshall-Ponting, A., Arayici, Y., \& Khosrowshahi, F. (2009). Towards implementation of building information modelling in the construction industry. Training and Education.

McGraw Hill Construction. (2012). The Business Value of BIM in North America. En SmartMarket Report.

Miettinen, R., \& Paavola, S. (2014). Beyond the BIM utopia: Approaches to the development and implementation of building information modeling. Automation in Construction. https://doi.org/10.1016/j.autcon.2014.03.009

Muñoz, S. (s.f.). Openbim, estandarizar para afrontar los nuevos retos del mercado. BuildingSMART Spanish.

Plebankiewicz, E. (2018). Model of predicting cost overrun in construction projects. Sustainability (Switzerland). https://doi.org/10.3390/su10124387

Porwal, A., \& Hewage, K. N. (2013). Building Information Modeling (BIM) partnering framework for public construction projects. Automation in Construction. https://doi.org/10.1016/j.autcon.2012.12.004

Rogério dos Santos Alves; Alex Soares de Souza, et all. (2014). BIM and Construction Management - Proven tools, methods, and workflows. En Igarss 2014.

Sabol, L. (2008). Building Information Modeling \& Facility Management. En Design + Construction Strategies.

Son, H., Lee, S., \& Kim, C. (2015). What drives the adoption of building information modeling 
in design organizations? An empirical investigation of the antecedents affecting architects' behavioral intentions. Automation in Construction. https://doi.org/10.1016/j.autcon.2014.10.012

Succar, B. (2009). Building information modelling framework: A research and delivery foundation for industry stakeholders. Automation in Construction. https://doi.org/10.1016/j.autcon.2008.10.003

Zhang, X., Azhar, S., Nadeem, A., \& Khalfan, M. (2018). Using Building Information Modelling to achieve Lean principles by improving efficiency of work teams. International Journal of Construction Management. https://doi.org/10.1080/15623599.2017.1382083 